\title{
STELLAR DISTANCES BY THE BAADE-WESSELINK METHOD
}

\author{
G. P. DI BENEDETTO \\ Istituto di Fisica Cosmica del CNR, Milano, Italy
}

\begin{abstract}
Galactic and extragalactic distances to Cepheid variable stars according to a modern realization of the geometric Baade-Wesselink method are presented. Reliable results as accurate as few percent are currently achievable, allowing the cosmic distance scale calibration to be significantly improved with respect to previous calibrations.
\end{abstract}

\section{Introduction}

Modern calibrations of stellar surface brightness enable reliable and accurate apparent angular diameters to be inferred for stars from A to $\mathrm{M}$ spectral type by applying high-precision photometry in the magnitude-color combination $(V, V-K)$ suitable to minimize biasing effects such as reddening and metallicity. These distance-independent angular sizes can be matched with the distance-dependent linear radii achievable from radial velocity measurements to provide stellar distances according to the geometric BW method. The color domain sampled by the surface brightness scale includes several important distance markers of major cosmological concern. In this contribution I shall outline the implementation of the BW method for Cepheid variables, being these stars the most relevant standard candles for the cosmic distance scale calibration.

\section{Calibrations by interferometric and spectroscopic techniques}

BW results are known to depend crucially on the quality of the empirical surface brightness-color SC correlations available for inferring stellar sizes and then on the choice of the magnitude-color combination adopted $a b i n i$ tio to calibrate most of potential biasing effects. This is notably important

T.R. Bedding et al. (eds.),

Fundamental Stellar Properties: The Interaction between Observation and Theory, 25-28.

(C) 1997 IAU. Printed in the Netherlands. 
for Cepheid variable stars, since variations in gravity and/or microturbulence along their pulsation cycle can destroy the well defined single-valued SC correlation required by any BW realization (Gautschy 1987). The modern Michelson stellar interferometry has recently provided angular diameters of non variable stars as accurate as $5 \%$ or less (Davis and Tango 1986; Di Benedetto and Rabbia 1987; Mozurkewich et al. 1991) enabling now a deeper investigation and fundamental calibration of the SC correlations. By using these data, a much tighter correlation can be found for the visual surface brightness $S_{V}$ as a function of the infrared color $(\mathrm{V}-\mathrm{K})$, rather than of other current optical colors, and reliable and accurate angular sizes have been predicted through the almost ideal magnitude-color combination $(V, V-K)$ (Di Benedetto 1993). The same calibration has been also applied for representing Cepheid angular sizes (Di Benedetto 1994). Fig. 1 shows the most recent zero-point calibration of the SC correlation over the Cepheid color domain. A slope of $(1.30 \pm 0.01)$ has been derived according to radial displacement data of the cluster Cepheid U Sgr (Welch 1994) and found to be in fairly good agreement with that of non variable supergiants, giving evidence for a likely constant slope representing the correlation of all Cepheids. The zero-point is obtained by averaging the most accurate data of practically unreddened dwarfs and giants. But there appears clear evidence for several other stars, notably 6 supergiants, to be well represented by the same absolute calibration. Then, the SC correlation representing photospheric size variations of galactic Cepheids is likely to be:

$$
S_{V}=V_{0}+5 \log (\Phi / \mathrm{mas})=2.762+1.30(V-K)_{0},
$$

where the unreddened photometry is in the Johnson magnitude system. The Eq. (1) also allows average angular diameters to be derived according to the intensity mean magnitudes of only two phase points out of the whole cycle with results largely insensitive to reddening and metallicity (Di Benedetto 1994, 1995).

The correlation currently applied for inferring linear radii of Cepheids takes the form of a suitably calibrated period-radius PR relation, where the potentially very accurate period $\mathrm{P}$ is the current observable. The most recent realization of the spectroscopic BW technique has provided calibrating linear radii of galactic Cepheids by using radial displacement data along with the infrared photometry less sensitive to limiting factors critically affecting optical BW radii (Laney and Stobie 1995). The corresponding PR relation will be adopted below as the most reliable ridge-line correlation. It allows the mean linear radius (in solar units) of a galactic Cepheid to be derived according to:

$$
5 \log \langle R\rangle=5.355+3.75 \log (P / \text { days })
$$


Because of its geometrical nature, the $\mathrm{PR}$ relation is expected to be largely independent of metallicity effects.

\section{BW distances to Cepheid variable stars}

The angular and linear sizes achievable by Eq.s (1) and (2), respectively, can be combined to yield BW distance moduli to any Cepheid of known $V, K$ photometry and period $P$ according to the following observational relationship:

$$
\mu_{0}=\langle V\rangle+3.75 \log P-1.30(\langle V\rangle-\langle K\rangle)+2.434+0.18 A_{V}
$$

where intensity mean magnitudes are reported now along with an overall absorption term due to interstellar reddening. The small formal errors affecting the above calibrations would lead to individual BW distance moduli as accurate as $0.04 \mathrm{mag}$ or $2 \%$ in distance. However, both the reliability and uncertainty in using Eq. (3) should be critically checked by comparing the results with the calibrating distances to cluster Cepheids well determined by the ZAMS fitting approach. Fig. 2 shows such comparison for 25 ZAMS calibrators with currently published $P, V, K$ and absorption data. I find that 20 of these stars show an average residual $(\mathrm{BW}-\mathrm{ZAMS})=-(0.01 \pm 0.03) \mathrm{mag}$ with a scatter $\mathrm{SD}= \pm 0.12 \mathrm{mag}$, whereas the remaining 5 stars deviate by up to 5 SD. By removing these 5 discordant stars inducing significant systematic errors, the overall uncertainty on the absolute galactic distance scale can be set to the value of $\pm 0.04 \mathrm{mag}$, i.e. a factor two smaller than that currently quoted for the Pleiades distance modulus which limits the ZAMS calibration.

The Eq. (3) might also be applied in a straightforward manner to obtain distances to any set of extragalactic Cepheids with $P, V, K$ and absorption data. However, for more accurate individual distances, a composite ad hoc PR relation has to be recalibrated by using the available sets of extragalactic Cepheids in the Magellanic Clouds (LMC and SMC) (Di Benedetto 1994). According to the most recent composite fit, the overall PR slope in Eq.s (2) and (3) decreases to the value 3.65; their zero-points increase to 5.472 and 2.551 , respectively, leading to the BW distances of $d(L M C)=(52.0 \pm 0.6) \mathrm{kpc}$ and $d(S M C)=(63.2 \pm 0.7) \mathrm{kpc}$ with uncertainties not including the above contribution of $2 \%$ due to the absolute distance scale calibration. Strong support to reliability of the actual BW results comes from the distance to LMC itself. Its value compares remarkably well with that measured by the geometric expansion parallax of the SN1987A in LMC via HST observations, given by $d(S N 1987 A)=(51.1 \pm 1.5) \mathrm{kpc}$ (Panagia et al. 1996).

The actual BW approach can be readily extended for including either the Johnson-Cousins color relevant in the HST observations of Cepheids, 


\section{References}

Davis, J., Tango, W. J.: 1986, Nature 323,234

Di Benedetto, G. P., Rabbia Y.: 1987, Astron. Astrophys. 188, 114

Di Benedetto, G. P.: 1993, Astron. Astrophys. 270, 315

Di Benedetto, G. P.: 1994, Astron. Astrophys. 285,819

Di Benedetto, G. P. : 1995, Ap. J. 452, 195

Gautschy, A.: 1987, Vistas in Astron. 30, 197

Laney, C. D., Stobie, R. S.: 1995, Mon. Not. R. Astr. Soc. 274, 337

Mozurkewich et al.: 1991, Astron. J. 101, 2207

Panagia et al.: 1996, in The Extragalactic Distance Scale, STScI, May Symp., Baltimore (in press)

Welch D. L.: 1994, Astron. J. 108, 1421

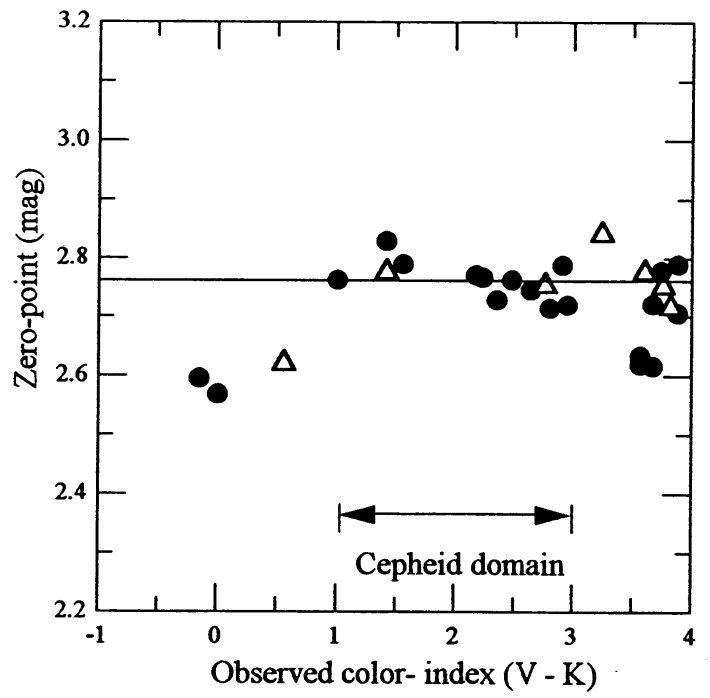

Fig. 1. Zero-point calibration of visual surface brightness by angular diameter measurements of non variable stars. Slope of linear SC correlation: 1.30 . Solid circles: dwarfs and giants. Open triangles: supergiants

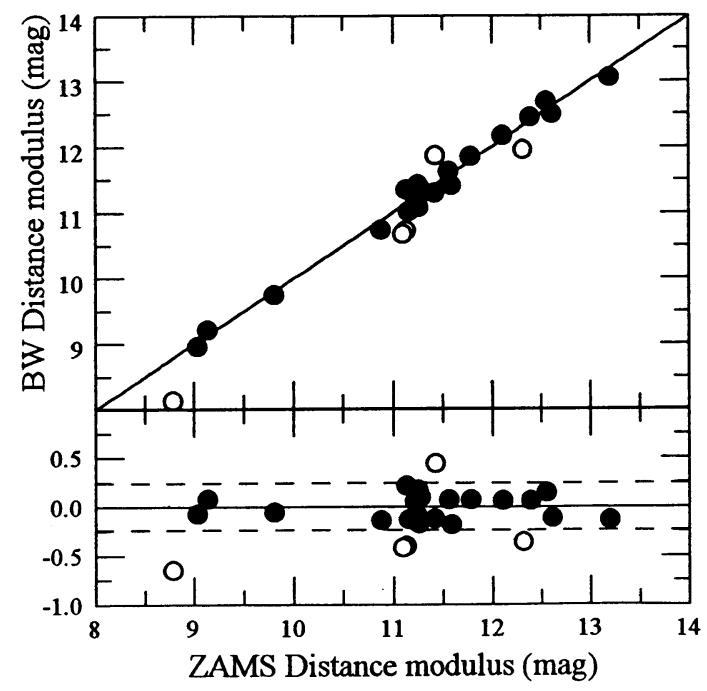

Fig. 2. BW vs. ZAMS distances of Cepheids in clusters. Solid line: locus of equal distances. Dashed lines are drawn at $\pm 0.24 \mathrm{mag}$ (2-sigma scatter). Open circles are stars falling more than 3 sigmas away from solid line.

Discussion of this paper appears at the end of these Proceedings. 